\title{
Refinements of Jordan's inequality
}

Meng-Kuang Kuo

Correspondence: kuo53@seed.net. tw

Center of General Education, JenTeh Junior College of Medicine, Nursing and Management, No. 799 Sha-Luen Hu Xi-Zhou Li HouLoung Town, Miaoli County,

Republic of China

\begin{abstract}
A method of sharpening Jordan's inequality proposed by Li-Li would be improved. Increasing lower bounds and decreasing upper bounds for strengthened Jordan's inequality can be constructed and the errors of lower-upper bounds for strengthened Jordan's inequality can be estimated.
\end{abstract}

(2010) Mathematics Subject Classification: 26D05; $26 \mathrm{D} 15$.

Keywords: Jordan?'?s inequality, lower bound, upper bound

\section{Introduction}

The well-known Jordan's inequality [1, p. 33] reads that

$$
\frac{2}{\pi} \leq \frac{\sin x}{x}<1, \quad 0<x \leq \frac{\pi}{2}
$$

with equality holds if and only if $x=\pi / 2$. This inequality plays an important role in many areas of pure and applied mathematics. Jordan's inequality (1.1) has been refined, generalized, and applied by many mathematicians. It was first extended to the following:

$$
\frac{\sin x}{x} \geq \frac{2}{\pi}+\frac{1}{12 \pi}\left(\pi^{2}-4 x^{2}\right), \quad 0<x \leq \frac{\pi}{2},
$$

and then, it was further refined to inequality (1.3),

$$
\frac{\sin x}{x} \geq \frac{2}{\pi}+\frac{1}{\pi^{3}}\left(\pi^{2}-4 x^{2}\right), \quad 0<x \leq \frac{\pi}{2} .
$$

For detailed information, please refer to the expository and survey articles [2] and related references therein.

In [3, Theorem 2.1] or [2, (2.26)], a new method of sharpening Jordan's inequality was established by Li-Li which shows that one can obtain strengthened Jordan's inequalities from old ones. This result may be stated as follows.

Theorem 1.1 (Li-Li) Let $g:[0, \pi / 2] \rightarrow[0,1]$ be a continuous function. If

$$
\frac{\sin x}{x} \geq g(x), \quad 0<x \leq \frac{\pi}{2}
$$

then the double inequality

$$
\frac{2}{\pi}-h\left(\frac{\pi}{2}\right)+h(x) \leq \frac{\sin x}{x} \leq 1+h(x), \quad 0<x \leq \frac{\pi}{2},
$$


holds with equality if and only if $x=\pi / 2$, where

$$
h(x)=-\int_{0}^{x} \frac{1}{u^{2}} \int_{0}^{u} v^{2} g(v) \mathrm{d} v \mathrm{~d} u, \quad 0 \leq x \leq \frac{\pi}{2} .
$$

However, the new ones are not always stronger than the old ones. For example, by taking $g(x)$ as the function in the right-hand side of (1.3), it was obtained that

$$
\frac{\sin x}{x} \geq \frac{2}{\pi}+\frac{1}{10 \pi}\left(\pi^{2}-4 x^{2}\right)+\frac{1}{80 \pi^{3}}\left(\pi^{2}-4 x^{2}\right)^{2}, \quad 0<x \leq \frac{\pi}{2} .
$$

The right-hand side inequality in (1.3) is slightly stronger than the right-hand side inequality in (1.7) at $x=\left(\frac{5\left(\pi^{2}-8\right)}{4}\right)^{1 / 2}$ but less than it at $x=0$. These two lower bounds for $\sin x / x$ can not be included each other. In fact, this obstacle can be improved and we can obtain better results (see (2.9)). Moreover, we can estimate the errors of lower bounds and upper bounds for strengthened Jordan's inequality (see $(2.12))$.

Let $g:[0, \pi / 2] \rightarrow[0,1]$ be a continuous function. Define the following functions, $h_{g}$ $(x), L_{g}(x)$ and $U_{g}(x)$, on the interval $[0, \pi / 2]$ by

$$
\begin{aligned}
& h_{g}(x):=-\int_{0}^{x} \frac{1}{u^{2}} \int_{0}^{u} v^{2} g(v) \mathrm{d} v \mathrm{~d} u, \\
& L_{g}(x):=\frac{2}{\pi}-h_{g}\left(\frac{\pi}{2}\right)+h_{g}(x), \quad U_{g}(x):=1+h_{g}(x) .
\end{aligned}
$$

For a positive integer $m$, we define

$$
L_{g}^{m}(x):=L_{L_{g}^{m-1}}(x), \quad L_{g}^{1}(x):=L_{g}(x), \quad L_{g}^{0}(x):=g(x) .
$$

Corollary 2.2 leads us to know that the lower bounds $L_{g}^{m}(x)$ and upper bounds $U_{L_{g}^{m}}(x)$ for $\sin x / x$ are increasing and decreasing with respect to positive integers $m$, respectively, and we obtain the double inequality (2.9). Corollary 2.4 , equality (2.12), leads us to know that the errors of lower-upper bounds for strengthened Jordan's inequality can be estimated and we give two examples such as (2.14) and (2.16) to estimate errors.

\section{Lower-upper bounds for Jordan's inequality}

With the help of Theorem 1.1, we find the important relations among lower-upper bounds for strengthened Jordan's inequality.

Theorem 2.1 Let $g_{1}, g_{2}:[0, \pi / 2] \rightarrow[0,1]$ be continuous functions. If $g_{1}(x) \leq g_{2}(x), 0 \leq$ $x \leq \pi / 2$, then

$$
\begin{array}{ll}
L_{g_{1}}(x) \leq L_{g_{2}}(x), & 0 \leq x \leq \frac{\pi}{2} \\
U_{g_{1}}(x) \geq U_{g_{2}}(x), & 0 \leq x \leq \frac{\pi}{2},
\end{array}
$$


and

$$
\begin{aligned}
\sup _{0 \leq x \leq \pi / 2}\left(L_{g_{2}}(x)-L_{g_{1}}(x)\right) & =L_{g_{2}}(0)-L_{g_{1}}(0) \\
& =\int_{0}^{\pi / 2} \frac{1}{u^{2}} \int_{0}^{u} v^{2}\left(g_{2}(v)-g_{1}(v)\right) \mathrm{d} v \mathrm{~d} u, \\
\sup _{0 \leq x \leq \pi / 2}\left(U_{g_{1}}(x)-U_{g_{2}}(x)\right) & =U_{g_{1}}\left(\frac{\pi}{2}\right)-U_{g_{2}}\left(\frac{\pi}{2}\right) \\
& =\int_{0}^{\pi / 2} \frac{1}{u^{2}} \int_{0}^{u} v^{2}\left(g_{2}(v)-g_{1}(v)\right) \mathrm{d} v \mathrm{~d} u .
\end{aligned}
$$

Proof. Let $0 \leq x \leq \pi / 2$. It follows from (1.8) and (1.9) that we have

$$
\begin{aligned}
L_{g_{2}}(x)-L_{g_{1}}(x) & =-h_{g_{2}}\left(\frac{\pi}{2}\right)+h_{g_{1}}\left(\frac{\pi}{2}\right)+h_{g_{2}}(x)-h_{g_{1}}(x) \\
& =\int_{0}^{\pi / 2} \frac{1}{u^{2}} \int_{0}^{u} v^{2}\left(g_{2}(v)-g_{1}(v)\right) \mathrm{d} v \mathrm{~d} u \\
& -\int_{0}^{x} \frac{1}{u^{2}} \int_{0}^{u} v^{2}\left(g_{2}(v)-g_{1}(v)\right) \mathrm{d} v \mathrm{~d} u \\
& =\int_{x}^{\pi / 2} \frac{1}{u^{2}} \int_{0}^{u} v^{2}\left(g_{2}(v)-g_{1}(v)\right) \mathrm{d} v \mathrm{~d} u \geq 0
\end{aligned}
$$

and (2.1) is obtained. The last term of (2.5) leads us to know that $L_{g_{2}}(x)-L_{g_{1}}(x)$ is decreasing to 0 as $x$ approaches to $\pi / 2$ and its supremum occurs at $x=0$ so that we obtain (2.3).

$$
U_{g_{1}}(x)-U_{g_{2}}(x)=h_{g_{1}}(x)-h_{g_{2}}(x)=\int_{0}^{x} \frac{1}{u^{2}} \int_{0}^{u} v^{2}\left(g_{2}(v)-g_{1}(v)\right) \mathrm{d} v \mathrm{~d} u \geq 0 .
$$

(2.6) is nonnegative and increasing so that we obtain (2.2) and (2.4) and we complete the proof.

If we set $g_{2}(x)=\sin x / x$ in Theorem 2.1, then we would obtain important results in the following corollary.

Corollary 2.2 Let $g:[0, \pi / 2] \rightarrow[0,1]$ be a continuous function. if $\sin x / x \geq g(x)$ and $L_{g}(x) \geq g(x), 0 \leq x \leq \pi / 2$, then for all positive integers $m$,

$$
L_{g}^{m-1}(x) \leq L_{g}^{m}(x), \quad 0 \leq x \leq \frac{\pi}{2},
$$

and

$$
U_{L_{s}^{m-1}}(x) \geq U_{L_{s}^{m}}(x), \quad 0 \leq x \leq \frac{\pi}{2} .
$$


Moreover,

$$
L_{g}^{m}(x) \leq \frac{\sin x}{x} \leq U_{L_{g}^{m-1}}(x), \quad 0<x \leq \frac{\pi}{2}
$$

Proof. It follows from Theorem 2.1, if replacing $g_{1}(x)$ and $g_{2}(x)$ by $g(x)$ and $L_{g}(x)$, respectively, then we have $L_{g}(x) \leq L_{g}^{2}(x)$. Repeating (2.1) in this inequality, we get that $L_{g}^{m}(x)$ is increasing with respect to positive integers $m$. A similar way, repeating (2.2), we have that $U_{L_{g}^{m}}(x)$ is decreasing with respect to positive integers $m$. With the help of Theorem 1.1, we have $L_{g}(x) \leq \sin x / x \leq U_{g}(x)$. Replacing $g(x)$ by $L_{g}(x)$, we have $L_{g}^{2}(x) \leq \sin x / x \leq U_{L_{g}}(x)$. Repeating this method, we obtain (2.9) and we complete our proof.

The following lemma will be used in the next corollary.

Lemma 2.3 Let $g(x)=\sin x / x, 0<x \leq \pi / 2$ and $g(0)=1$. Then

$$
\begin{array}{ll}
L_{g}(x)=\frac{\sin x}{x}, & 0<x \leq \frac{\pi}{2} \\
U_{g}(x)=\frac{\sin x}{x}, & 0<x \leq \frac{\pi}{2},
\end{array}
$$

$L_{g}(0)=1$ and $U_{g}(0)=1$.

Proof. For $0<x \leq \pi / 2$. It follows from elementary calculus and Fubini's Theorem that we have

$$
\begin{aligned}
\int_{0}^{x} \frac{1}{u^{2}} \int_{0}^{u} v^{2} \frac{\sin v}{v} \mathrm{~d} v \mathrm{~d} u & =\int_{0}^{x} v \sin v \int_{v}^{x} \frac{1}{u^{2}} \mathrm{~d} u \mathrm{~d} v \\
& =\int_{0}^{x} v \sin v\left(\frac{1}{v}-\frac{1}{x}\right) \mathrm{d} v=1-\frac{\sin x}{x}
\end{aligned}
$$

and

$$
\begin{aligned}
L_{g}(x) & =\frac{2}{\pi}+\int_{0}^{\pi / 2} \frac{1}{u^{2}} \int_{0}^{u} v^{2} \frac{\sin v}{v} \mathrm{~d} v \mathrm{~d} u-\int_{0}^{x} \frac{1}{u^{2}} \int_{0}^{u} v^{2} \frac{\sin v}{v} \mathrm{~d} v \mathrm{~d} u \\
& =\frac{2}{\pi}+\left(1-\frac{2}{\pi} \sin \frac{\pi}{2}\right)-\left(1-\frac{\sin x}{x}\right)=\frac{\sin x}{x}
\end{aligned}
$$

also we have

$$
U_{g}(x)=1-\int_{0}^{x} \frac{1}{u^{2}} \int_{0}^{u} v^{2} \frac{\sin v}{v} \mathrm{~d} v \mathrm{~d} u=\frac{\sin x}{x}
$$

By approximation, we set $L_{g}(0)=1$ and $U_{g}(0)=1$ and we get our desired results.

The errors of lower-upper bounds for strengthened Jordan's inequality can be estimated in the following corollary.

Corollary 2.4 Let $g:[0, \pi / 2] \rightarrow[0,1]$ be a continuous function. If

$$
\frac{\sin x}{x} \geq g(x), \quad 0<x \leq \frac{\pi}{2},
$$


then

$$
\begin{aligned}
\sup _{0 \leq x \leq \pi / 2}\left(\frac{\sin x}{x}-L_{g}(x)\right) & =1-\frac{2}{\pi}-\int_{0}^{\pi / 2} \frac{1}{u^{2}} \int_{0}^{u} v^{2} g(v) \mathrm{d} v \mathrm{~d} u \\
& =\sup _{0 \leq x \leq \pi / 2}\left(U_{g}(x)-\frac{\sin x}{x}\right) .
\end{aligned}
$$

Proof. It follows from Lemma 2.3 and equalities (2.3) and (2.4), if replacing $g_{1}(x)$ and $g_{2}(x)$ by $g(x)$ and $\sin x / x$, respectively, then we have

$$
\begin{aligned}
\sup _{0 \leq x \leq \pi / 2}\left(\frac{\sin x}{x}-L_{g}(x)\right) & =1-L_{g}(0)=1-\left(\frac{2}{\pi}-h_{g}\left(\frac{\pi}{2}\right)+h_{g}(0)\right) \\
& =1-\frac{2}{\pi}-\int_{0}^{\pi / 2} \frac{1}{u^{2}} \int_{0}^{u} v^{2} g(v) \mathrm{d} v \mathrm{~d} u, \\
\sup _{0 \leq x \leq \pi / 2}\left(U_{g}(x)-\frac{\sin x}{x}\right) & =U_{g}\left(\frac{\pi}{2}\right)-\frac{2}{\pi}=1+h_{g}\left(\frac{\pi}{2}\right)-\frac{2}{\pi} \\
& =1-\int_{0}^{\pi / 2} \frac{1}{u^{2}} \int_{0}^{u} v^{2} g(v) \mathrm{d} v \mathrm{~d} u-\frac{2}{\pi} .
\end{aligned}
$$

We give some examples of (2.9) and (2.12) here. Let $g(x)=\pi / 2$. It follows from (1.9) that $L_{g}(x)=\frac{2}{\pi}+\frac{\pi}{12}-\frac{1}{3 \pi} x^{2} \cdot L_{g}(x)-g(x)=\frac{\pi}{12}-\frac{1}{3 \pi} x^{2}$ is decreasing on $[0, \pi / 2]$ and $\inf _{0 \leq x \leq \pi / 2} L_{g}(x)-g(x)=L_{g}(\pi / 2)-g(\pi / 2)=0$, so that $L_{g}(x) \geq g(x)$. It follows from (1.1), $L_{g}$ $(x) \geq g(x)$ and choosing $m=2$ in (2.9) that we have $L_{g}^{2}(x) \leq \sin x / x \leq U_{L_{g}}(x)$,

$$
\frac{2}{\pi}+\frac{\pi}{12}+\frac{7 \pi^{3}}{2,880}-\left(\frac{1}{3 \pi}+\frac{\pi}{72}\right) x^{2}+\frac{1}{60 \pi} x^{4} \leq \frac{\sin x}{x} \leq 1-\left(\frac{1}{3 \pi}+\frac{\pi}{72}\right) x^{2}+\frac{1}{60 \pi} x^{4} .
$$

Moreover, by (2.12), we have

$$
\begin{aligned}
\sup _{0 \leq x \leq \pi / 2}\left(\frac{\sin x}{x}-L_{g}^{2}(x)\right) & =\sup _{0 \leq x \leq \pi / 2}\left(U_{L_{g}}(x)-\frac{\sin x}{x}\right) \\
& =1-\frac{2}{\pi}-\frac{\pi}{12}-\frac{7 \pi^{3}}{2,880} \approx 0.0262 .
\end{aligned}
$$

The lower bound of (2.13) is stronger than the lower bound of (1.2) and equal at $x=$ $\pi / 2$. But the lower bounds of (2.13) and (1.3) cannot be included each other. In this case, when $x \in[0, \beta]$, the left-hand side inequality in (2.13) is stronger than the righthand side inequality in (1.3) but less than it as $x$ is close to $\pi / 2$, where

$$
\begin{aligned}
\beta & =\left(\frac{5 \pi^{4}+120 \pi^{2}-1,440-2\left(\pi^{8}+120 \pi^{6}+2,160 \pi^{4}-86,400 \pi^{2}+518,400\right)^{1 / 2}}{12 \pi^{2}}\right)^{1 / 2} \\
& \approx 1.2 .
\end{aligned}
$$


For $L_{g}^{3}(x) \leq \sin x / x \leq U_{L_{g}^{2}}(x)$, we have

$$
\begin{aligned}
& \frac{2}{\pi}+\frac{\pi}{12}+\frac{7 \pi^{3}}{2,880}+\frac{31 \pi^{5}}{483,840}-\left(\frac{1}{3 \pi}+\frac{\pi}{72}+\frac{7 \pi^{3}}{17,280}\right) x^{2}+\left(\frac{1}{60 \pi}+\frac{\pi}{1,440}\right) x^{4}-\frac{1}{2,520 \pi} x^{6} \\
& \leq \frac{\sin x}{x} \leq 1-\left(\frac{1}{3 \pi}+\frac{\pi}{72}+\frac{7 \pi^{3}}{17,280}\right) x^{2}+\left(\frac{1}{60 \pi}+\frac{\pi}{1,440}\right) x^{4}-\frac{1}{2,520 \pi} x^{6},
\end{aligned}
$$

and

$$
\begin{aligned}
\sup _{0 \leq x \leq \pi / 2}\left(\frac{\sin x}{x}-L_{g}^{3}(x)\right) & =\sup _{0 \leq x \leq \pi / 2}\left(U_{L_{g}^{2}}(x)-\frac{\sin x}{x}\right) \\
& =1-\frac{2}{\pi}-\frac{\pi}{12}-\frac{7 \pi^{3}}{2,880}-\frac{31 \pi^{5}}{483,480} \approx 0.0066
\end{aligned}
$$

It is clear that the error in (2.16) is much smaller than that in (2.14), and it seems to us that $L_{g}^{m}(x)$ and $U_{L_{g}^{m}}(x)$ are convergent to $\sin x / x$ uniformly. However, we have not proved our conjecture yet.

\section{Acknowledgements}

The author expresses his sincere thanks to the referees for careful reading of the article and several helpful suggestions.

\section{Competing interests}

The author declares that he has no competing interests.

Received: 9 April 2011 Accepted: 5 December 2011 Published: 5 December 2011

\section{References}

1. Mitrinović, DS, Vasić, PM: Analytic Inequalities. Springer, New York (1970)

2. Qi, F, Niu, D-W, Guo, B-N: Refinements, generalizations, and applications of Jordan's inequality and related problems. J Inequal Appl 52 (2009). 2009(Article ID 271923)

3. Li, J-L, Li, Y-L: On the strengthened Jordan's inequality. J Inequal Appl 8 (2007). 2007(Article ID 74328)

doi:10.1186/1029-242X-2011-130

Cite this article as: Kuo: Refinements of Jordan's inequality. Journal of Inequalities and Applications 2011 2011:130.

\section{Submit your manuscript to a SpringerOpen ${ }^{\odot}$} journal and benefit from:

- Convenient online submission

Rigorous peer review

- Immediate publication on acceptance

- Open access: articles freely available online

- High visibility within the field

- Retaining the copyright to your article

Submit your next manuscript at $\boldsymbol{\nabla}$ springeropen.com 